\title{
Assessment of Resources for Physics Teaching in Nigerian Senior Secondary Schools
}

\author{
Akinbobola, Akinyemi Olufunminiyi, Ph.D*, Bada, Abiodun Adekunle, Ph.D \\ Department of Special Education and Curriculum Studies, Adeyemi College of Education, Ondo, Nigeria \\ *Corresponding Author: Akinbobola, Akinyemi Olufunminiyi, Department of Special Education and \\ Curriculum Studies, Adeyemi College of Education, Ondo, Nigeria
}

\begin{abstract}
The study assessed the resources for Physics teaching in public and private senior secondary schools in Ondo West Local Government Area of Ondo State, Nigeria. Descriptive survey design was adopted for the study. A total of 60 Physics teachers took part in the study. The instrument used for collecting data was Resources for Physics Teaching Questionnaire (RPTQ) with internal consistency of 0.81 using Cronbach Alpha. The results showed that, majority of the Physics teachers available in senior secondary schools in Ondo West Local Government Area of Ondo State, Nigeria are qualified to teach Physics but the numbers are not adequate. The results also indicated that, there are more qualified Physics teachers and laboratory facilities in public schools than private schools. Also, laboratory facilities for the teaching of Physics are not adequate in number for effective teaching of Physics. It is recommended among other that, more resources (human and non-human) should be provided by government, philanthropists and non-governmental organisations in order to boost effective teaching and learning of Physics in Nigeria.
\end{abstract}

Keywords: Resources, Physics Teaching, Public and Private Schools.

\section{INTRODUCTION}

Physics is the bedrock of science and technology because many of the tools on which the scientific and technological advancement depends are the direct products of Physics. Physics is therefore a core subject in the development of science and technology since it assists individuals to understand the rapidly technological changing society and it entails the essence of various concepts and phenomena (Akinbobola, 2015). The principles of Physics have been widely used for various economic, technological and scientific advancement, such as, in information and communication technology, which has reduced the world into a global village through the use of smart phones, computers, ipads and satellites. Also, the knowledge of Physics had led to sustainable development in the area of industrialization for improvement of material useful to the well-being of human race. Also, Physics education enables the students to acquire problem-solving and science process skills that will enhance proper critical thinking and inquiry that could help them to respond to widespread and radical changes in all facets of life (Akinbobola \& Bada, 2017).

Resources are very vital tools in education. They aid teaching and learning and include everything that provides information to the teacher as well as the learner. Resources include all forms of information that are used to promote, encourage, and enhance teaching and learning and learning activities (Akinbobola, 2006). With regards to education, resources means something that can be used to increase or enhance or improve, or implement educational programme. Babalola (1992) as cited by Akpochafo (2003) defines resources to include man, machine, students, materials controlled and coordinated to achieve better teaching. There are two types of resources namely human and nonhuman resources (Akinbobola, 2006). Hofstein and Mamlok-Naaman (2007) categorized resources required for science teaching to include human resources, material resources, library resources, and industrial and science resource centres. In order to ensure sustainable teaching-learning processes in Physics, it is important that human and non-human (materials, physical and financial) resources must be adequately provided.

Achimugu (1998) refers to human resources to mean human beings who directly or indirectly contribute to the achievement of science educational objectives. These include learners, laboratory 
technicians and the teachers. Material resources are structural facilities that are used to ensure effective teaching and learning, such as the classrooms, laboratories, tools, equipment, textual material, audio aids, visual aids and audio-visual aids. In this study, human resources are limited to Physics teachers while material resources are limited to laboratory facilities. Moorhead and Griffin (2002) states that, the success of any organization depends to a great extent on the status of the human resources because it coordinates both financial and physical resources for the purpose of achieving the organizational goals. Also, human resources could be categorized into teaching and non-teaching staff, and the learners. The role of teacher however is the most important. Without a well trained, strongly motivated, skilled and well supported teacher, the arch of excellent in senior secondary school Physics collapses. The teacher is the keystone of quality education. Molla (2012) characterizes an effective teacher as one who matches the strategies to the students learning styles in his effort to promote creativity and innovations.

Effective teaching and learning should ensure a coherent preparation of individuals for happy and useful living in the society. This can only be accomplished within an instructional frame work of a teaching methods and strategies. Factors to be considered in the choice of a strategy or method of teaching include:

- The age, mental ability, physical development, maturity, educational background and the entry behaviour of the learner.

- The language competence in terms of communication skills (oral and written expression) of the learners.

- Time allotted for each lesson or period per week.

- Adequacy, appropriateness and relevance of instructional materials for proper illustration and explanation of basic concepts, principles and events.

- Sustainability of the learning environments which include physical infrastructures such as library, laboratories, classroom and field/court.

- Objectives of the lesson which determine the mode of assessment of learning outcomes.

- Interest and competence of the teacher as regards to the effectiveness of using the method.

- The purpose, shape and nature of instruction determine the method of teaching to be employed (Oyekan \& Akinbobola, 2017).

A Physics laboratory is a place where research and experiment are carried out, and all the tools, materials and equipment are kept for scientific investigation (Akinbobola, 2015). The use of Physics laboratory helps to integrate theory and practical work in Physics teaching. According to Olufunke (2012), advantage of using Physics laboratory include:

- Avenue for experimentation through practical work

- Display physical information

- Removing abstractness and increase effective teaching and learning.

- Poor storage of physical materials for easy access.

Based on the advantage of Physics laboratory, it is expected that teaching and learning of Physics, with Physics laboratory may help to reduce abstract nature of the subject and also help the students to overcome many misconceptions about the physical world that they have developed before undertaking formal instruction in the subject.

The use of aids in teaching is of importance as they help to stimulate learners' interest and promote understanding. According to Ogweno (2015), the teaching and learning of science which is a practical course requires practical laboratory activities because experiment is the hall mark of conducting practical classes. Thus, even when materials are within reach, they may refuse to use them. The condition under which many teachers function does not engender any enthusiasm for practical work. The class size especially in urban school in Nigeria is getting larger. Hence, the importance of adequate and well utilize laboratory cannot be over emphasize for effective Physics teaching. The National Research Council (NRS, 2000), states that, inquiry-type laboratories have the potential to 
develop students' abilities and science process skills which enhance hands-on and minds-on activities. Laboratory activities according to Hofstein and Lunetta (2004) is a means of learning with understanding, and a process of constructing knowledge by active learning or learning by ding. Also, quality and meaningful learning is possible in the laboratory if students are given opportunity to manipulate tools, materials and equipment in order to be able to construct their knowledge in related scientific concept and phenomena. Hence, laboratory activities motivate students by stimulating interest and attitude, teach laboratory skills, assist concept development and acquisition, encourage social skill development and inculcate scientific attitudes (Owoeye \& Yara, 2011).

Resource utilization is the process of managing and organizing resources for learning and teaching (Sally, 2010). Resource utilization deals with the extent to which materials, tools and equipment are effectively used. When the tools, equipment and materials are maximally used, they are effectively utilized and when they are not maximally used, it is said to be under-utilized. When there is so much pressure on the use of tools, materials and equipment, it may result to over utilization which could lead to break down of such tools, materials and equipment (Tobin, 1990). Resource materials utilization during practical lesson develops in the learner the manipulative skills, communicative skills, creative skills, organisational skills and acquisitive skills (Hofstein, Navon, Kipnis \& MamlokNaaman, 2005). Maintenance of tools, materials and equipment in order to prolong their life span is also an aspect of resource utilization. Maintenance involves all activities that are put in place to keep and restore the condition of materials, tools and equipment so as to enable them to be in a good working condition for as long as possible. When activities such as greasing, servicing and repairs are put in place to restore or keep the component of an equipment, the equipment is being maintained. Maintenance prevents deterioration and also weeds out obsolete equipment which no longer serve the required function. According to Krajcik, Mamlok and Hug (2001), objective of maintenance of facilities include:

- Ensuring that equipment, tools and materials are always available to provide services for maximum benefits to staff and students.

- Protecting the operation of personnel and save facilities

- Ensuring operational readiness of tools, equipment and materials for continuous service so as to reduce losses which may result from down time

- Ensuring the use of the equipment, tools and materials for maximum benefit.

Maintenance could be routine ongoing activities such as weekly, daily or monthly cleaning of laboratory tools, equipment and materials. It could be periodic activities such as inspection and lubrication of parts of equipment to ensure continued working condition or corrective maintenance which includes activities carried out to fix back a bad tool or equipment (Ogweno, 2015). Inability to appropriately manage resource in the laboratory is a sign of poor management. Akinbobola (2006), states that, maintenance culture is very poor in Nigerian schools. The tools, materials and equipment are laying waste due to breakdown, and some are forced to breakdown by dust and cobwebs due to negligence and lack of care. Hence, this study assessed the current status of resources for Physics teaching in public and private senior secondary schools in Ondo West Local Government Area of Ondo State, Nigeria.

\section{Statement of the Problem}

A survey of senior secondary school Physics laboratories in Nigeria reveals that many of them are underfunded with outdated equipment, materials and tools. The classroom experience shows that a large number of the senior secondary school Physics students face considerable difficulty in appreciating and learning Physics concepts in a meaningful way, especially in laboratory activities. This is a clear reflection in their poor ability to solve simple day to day problems, make predictions in given situations and have poor ability to apply Physics concepts to explain ordinary natural phenomena. In most senior secondary schools in Nigeria, there are no adequate tools, materials and equipment for effective delivery of Physics education instructions. In some cases, the population of students may be greater than the available laboratory facilities. In a situation where laboratory facilities are available for learning in schools, it is discovered that they are not well maintained and utilized. This situation does not enhance effective learning of Physics. Also, since the population of 
students has continue to grow every year at the expense of available laboratory facilities, and the government has failed to expand or put in place new laboratory facilities, the existing laboratory facilities are been over utilized because of the pressure on them. Therefore, the laboratory activities do not match government provision of laboratory tools, materials and equipment possibly due to the condition of the national economy which continues to deteriorate. This might lead to poor academic performance of students in Physics.

\subsection{Purpose of the Study}

The purpose of the study is to assess the status of resources for Physics teaching in Ondo West Local Government Area of Ondo State, Nigeria.

\subsection{Research Questions}

- Are the teachers in private and public senior secondary schools in Ondo West Local Government Area in Ondo State of Nigeria available for the teaching of Physics?

- Are the laboratory facilities available in private and public senior secondary schools in Ondo West Local Government Area in Ondo state of Nigeria?

\subsection{Hypotheses}

$>$ There is no significant difference between the number of qualified Physics teacher in private and public senior secondary schools in Ondo West Local Government Area in Ondo State of Nigeria.

$>$ There is no significant difference between the quantity of available laboratory facilities for teaching Physics in private and public senior secondary schools in Ondo West Local Government Area in Ondo State of Nigeria.

$>$ There is no significant difference between the utilization of the available laboratory facilities for the teaching of Physics in private and public senior secondary schools in Ondo West Local Government Area in Ondo State of Nigeria.

\section{RESEARCH METHOD}

Descriptive survey design was adopted for the study. The population of the study consisted of 60 Physics teachers (40 from public and 20 from private senior secondary schools) in Ondo West Local Government Area of Ondo State, Nigeria. All the Physics teachers were used for the study because of the few numbers of them involved. The instrument used in collecting data for the study was Resources for Physics Teaching Questionnaire (RPTQ). The RPTQ was designed by the researchers and it consisted of two sections. Section A consisted of demographic data on human resources such as teacher's age, gender, qualification, experience and the number of Physics teachers available in the school. Section B comprised of structured information on the availability of and utilization of laboratory facilities.

The instrument was validated by two professional Physics teachers. The RPTQ was trial tested with Physics teachers who were not part of the main study. The data collected from RPTQ were analysed using Cronbach Alpha. The result showed an internal consistency of 0.81. The instrument was then administered to the respondents. The research questions were analysed using frequency count (f) and percentage $(\%)$ while the hypotheses were test with Ch-square $\left(\mathrm{x}^{2}\right)$ at 0.05 level of significance.

\section{RESULTS AND DisCUSSION}

Research Question 1: Are the teachers in private and public senior secondary schools in Ondo West Local Government Area in Ondo State of Nigeria available for the teaching of Physics?

Table1 showed the frequency and percentage of the available teachers by qualification in the selected schools. Table 1 also showed that, Physics teachers in public senior secondary schools in Ondo West Local Government Area in Ondo State of Nigeria consisted of NCE holders (0\%), BSc(Ed)/B.Ed. $(8 \%)$, HND (5\%), OND (0\%), BSc. (2.5\%), M.Sc.(7.5\%) and holders of M.Ed./M.Sc.Ed. (5\%) and Ph.D.(0\%) respectively, while private senior secondary schools have NCE (10\%), OND (5\%), HND (10\%), B.Sc.Ed./B.Ed. (5\%), MSc. (5\%) and holders of Med./M.Sc.Ed. (5\%) and Ph.D (0\%) respectively.

The analysis as shown in Table 1. 
Table1: Frequency distribution and percentage of available Physics teachers

\begin{tabular}{|l|l|l|l|l|l|l|}
\hline \multicolumn{3}{|c|}{ Public } & \multicolumn{3}{c|}{ Private } \\
\hline Qualification & $\begin{array}{l}\text { Number of } \\
\text { available } \\
\text { Physics teachers }\end{array}$ & Qualified & $\begin{array}{l}\text { Not } \\
\text { Qualified }\end{array}$ & $\begin{array}{l}\text { Number of } \\
\text { available Physics } \\
\text { teachers }\end{array}$ & Qualified & $\begin{array}{l}\text { Not } \\
\text { Qualified }\end{array}$ \\
\hline OND & & & $0(0 \%)$ & 1 & & $1(5 \%)$ \\
\hline NCE & & & $0(0 \%)$ & 2 & & $2(10 \%)$ \\
\hline HND & 2 & & $2(5 \%)$ & 2 & & $2(10 \%)$ \\
\hline B.Sc. & 1 & & $1(2.5 \%)$ & 3 & & $3(15 \%)$ \\
\hline B.Sc.Ed/B.Ed. & 32 & $32(80 \%)$ & & 10 & $10(50 \%)$ & \\
\hline M.Sc. & 3 & & $3(7.5 \%)$ & 1 & $1(5 \%)$ & \\
\hline M.Ed./M.Sc.Ed. & 2 & $2(5 \%)$ & & 1 & $1(5 \%)$ & \\
\hline Ph.D & & & & & & \\
\hline Total Available & 40 & & & 20 & & \\
\hline Expected & 56 & & & 30 & & \\
\hline
\end{tabular}

Note:

- Qualified teachers are those with at least degrees in Physics education B.Sc.(Ed)/B.Ed. Physics or B.Sc. Physics with PostGraduate Diploma in Education (PGDE) and M.Ed./M.Sc. or M.Sc. Physics with PGDE.

- Not qualified teachers are those with Higher National Diploma (HND), Ordinary National Diploma (OND), Nigeria Certificate in Education (NCE), B.Sc. and M.Sc. Physics.

Table 1 also indicated that, majority of the Physics teachers $85 \%$ and $55 \%$ in public and private senior secondary schools respectively in Ondo West Local Government Area in Ondo State of Nigeria are qualified to teach Physics.The results is in agreement with the findings of Akinbobola (2006) that majority of the Physics teachers in our senior secondary schools are qualified to teach the subjects. However, each of the school in 40 public and 20 private senior secondary schools in Ondo West Local Government Area in Ondo State of Nigeria is expected to have at least one Physics teacher. This means that $40(71.43 \%)$ out of 56 public senior secondary schools and $20(66.6 \%)$ out of 30 private senior secondary schools have Physics teachers. This implies that, the numbers of Physics teachers available in Ondo West Local Government Area in Ondo State of Nigeria are not adequate in both private and public senior secondary schools.

Research Question 2: Are the laboratory facilities available in private and public senior secondary schools in Ondo West Local Government Area in Ondo State of Nigeria?

Table2: Availability and adequacy of the laboratory facilities for Physics teaching in public and private senior secondary schools

\begin{tabular}{|l|l|l|l|l|l|}
\hline \multirow{2}{*}{ SN } & \multirow{2}{*}{ Laboratory facilities } & \multicolumn{2}{c|}{ Public schools } & \multicolumn{2}{c|}{ Private schools } \\
\cline { 3 - 6 } & & $\begin{array}{l}\text { Availability } \\
(\mathbf{A V})\end{array}$ & $\begin{array}{l}\text { Utilization } \\
(\text { UT })\end{array}$ & $\begin{array}{l}\text { Availability } \\
(\mathbf{A V})\end{array}$ & $\begin{array}{l}\text { Utilization } \\
\text { (UT) }\end{array}$ \\
\hline 1 & Avometer & $26(65 \%)$ & $26(65 \%)$ & $7(35 \%)$ & $7(35 \%)$ \\
\hline 2 & Ammeter0-3ADC & $35(87.5 \%)$ & $35(87.5 \%)$ & $18(90 \%)$ & $18(90 \%)$ \\
\hline 3 & $1 / 2$ Amp fuse & $35(87.5 \%)$ & $22(55 \%)$ & $10(50 \%)$ & $10(50 \%)$ \\
\hline 4 & Accumulator lead acid car type & $22(55 \%)$ & $16(40 \%)$ & $15(75 \%)$ & $15(75 \%)$ \\
\hline 5 & Asbestos sheet 20cm x20cm & $26(65 \%)$ & $26(65 \%)$ & $6(30 \%)$ & $6(30 \%)$ \\
\hline 6 & Aluminum scale pans & $22(55 \%)$ & $22(55 \%)$ & $5(25 \%)$ & $3(15 \%)$ \\
\hline 7 & Balance beam type & $35(87.5 \%)$ & $35(85.5 \%)$ & $17(85 \%)$ & $16(80 \%)$ \\
\hline 8 & Balance lever type & $30(70 \%)$ & $29(72.5 \%)$ & $14(80 \%)$ & $13(65 \%)$ \\
\hline 9 & Box of weight & $32(80 \%)$ & $32(80 \%)$ & $17(85 \%)$ & $17(85 \%)$ \\
\hline 10 & Ballons & $20(50 \%)$ & $18(45 \%)$ & $5(25 \%)$ & $6(30 \%)$ \\
\hline 11 & Boiling flask 250 cc & $34(85 \%)$ & $32(80 \%)$ & $13(65 \%)$ & $13(65 \%)$ \\
\hline 12 & Burettes 50x0.1cm & $38(95 \%)$ & $38(95 \%)$ & $19(95 \%)$ & $18(90 \%)$ \\
\hline 13 & Ball and Rings & $32(80 \%)$ & $31(77.5 \%)$ & $13(65 \%)$ & $13(65 \%)$ \\
\hline 14 & Bimetallic strips & $25(62.5 \%)$ & $24(60 \%)$ & $5(25 \%)$ & $5(25 \%)$ \\
\hline 15 & Bar and gauge apparatus & $17(42.5 \%)$ & $15(37.5 \%)$ & $10(50 \%)$ & $10(50 \%)$ \\
\hline 16 & Breaking bars-cast iron & $20(50 \%)$ & $15(37.5 \%)$ & $1(5 \%)$ & $1(5 \%)$ \\
\hline 17 & Barometer fortins & $21(52.5 \%)$ & $21(52.5 \%)$ & $10(50 \%)$ & $10(50 \%)$ \\
\hline 18 & Bulbs 2.5V,0.3A & $34(85 \%)$ & $34(85 \%)$ & $17(85 \%)$ & $16(80 \%)$ \\
\hline
\end{tabular}




\begin{tabular}{|c|c|c|c|c|c|}
\hline 19 & Bulbs holders & $31(77.5 \%)$ & $31(77.5 \%)$ & $19(95 \%)$ & $17(85 \%)$ \\
\hline 20 & Beakers & $38(95 \%)$ & $38(95 \%)$ & $19(95 \%)$ & $18(90 \%)$ \\
\hline 21 & Cork assorted box-wood & $26(65 \%)$ & $26(65 \%)$ & $13(65 \%)$ & $13(65 \%)$ \\
\hline 22 & Cylinder and bucket & $33(82.5 \%)$ & $33(82.5)$ & $16(80 \%)$ & $16(80 \%)$ \\
\hline 23 & $\begin{array}{l}\begin{array}{l}\text { Capillary tubes length } 140 \mathrm{~cm} \text { assorted } \\
\text { bores }\end{array} \\
\end{array}$ & $24(60 \%)$ & $23(57.5 \%)$ & $10(50 \%)$ & $10(50 \%)$ \\
\hline 24 & Calipers sliding venire & $30(75 \%)$ & $30(75 \%)$ & $12(60 \%)$ & $12(60 \%)$ \\
\hline 25 & Copper calorimeter & $34(85 \%)$ & $30(75 \%)$ & $13(65 \%)$ & $10(50 \%)$ \\
\hline 26 & Compass mariners & $14(35 \%)$ & $14(35 \%)$ & $4(20 \%)$ & $4(20 \%)$ \\
\hline 27 & Compass plotting & $31(77.5 \%)$ & $18(45 \%)$ & $5(25 \%)$ & $5(25 \%)$ \\
\hline 28 & Crocodile clips & $36(90 \%)$ & $32(80 \%)$ & $18(90 \%)$ & $17(85 \%)$ \\
\hline 29 & Cells-Daniel complete & $22(55 \%)$ & $18(45 \%)$ & $13(65 \%)$ & $13(65 \%)$ \\
\hline 30 & $\mathrm{Ni}-\mathrm{Fe}$ Alkaline & $21(52.5 \%)$ & $22(55 \%)$ & $10(50 \%)$ & $10(50 \%)$ \\
\hline 31 & Leclanche wet type & $23(57.5 \%)$ & $22(55 \%)$ & $10(50 \%)$ & $10(50 \%)$ \\
\hline 32 & Cork boners & $20(50 \%)$ & $22(55 \%)$ & $5(25 \%)$ & $3(15 \%)$ \\
\hline 33 & Density bottles & $31(77.5 \%)$ & $30(75 \%)$ & $5(25 \%)$ & $4(20 \%)$ \\
\hline 34 & Dip circle & $11(27.5 \%)$ & $7(17.5 \%)$ & $1(5 \%)$ & $1(5 \%)$ \\
\hline 35 & Dip needles & $14(35 \%)$ & $11(27.5 \%$ & $1(5 \%)$ & $1(5 \%)$ \\
\hline 37 & Electroscope simple flat type (complete) & $13(32.5 \%)$ & $11(27.5 \%)$ & $1(5 \%)$ & $1(5 \%)$ \\
\hline 38 & Electric bells & $18(45 \%)$ & $18(45 \%)$ & $4(20 \%)$ & $4(20 \%)$ \\
\hline 39 & Electric motor(1 watt) $1.5-6 \mathrm{v}$ & $20(50 \%)$ & $20(50 \%)$ & $3(15 \%)$ & $3(15 \%)$ \\
\hline 40 & Electric bell jars & $20(50 \%)$ & $18(45 \%)$ & $2(10 \%)$ & $2(10 \%)$ \\
\hline 41 & Fur & $5(12.5 \%)$ & $6(15 \%)$ & $1(5 \%)$ & $1(5 \%)$ \\
\hline 42 & Flame spectra kit & $10(25 \%)$ & $10(25 \%)$ & $5(25 \%)$ & $4(20 \%)$ \\
\hline 43 & Fluid pressure apparatus & $11(27.5 \%)$ & $10(25 \%)$ & $1(5 \%)$ & $1(5 \%)$ \\
\hline 44 & Glass tubes claim & $12(30 \%)$ & $10(25 \%)$ & $10(50 \%)$ & $10(50 \%)$ \\
\hline 45 & Glass prism & $26(65 \%)$ & $25(62.5 \%)$ & $14(80 \%)$ & $14(80 \%)$ \\
\hline 46 & Glass semi-circular block & $20(50 \%)$ & $20(50 \%)$ & $11(55 \%)$ & $10(50 \%)$ \\
\hline 47 & Glass cube hallow & $7(17.5 \%)$ & $4(10 \%)$ & $3(15 \%)$ & $3(15 \%)$ \\
\hline 48 & Glass rods & $23(57.5 \%)$ & $23(57.5 \%)$ & $11(55 \%)$ & $11(55 \%)$ \\
\hline 49 & Galvanometer & $28(70 \%)$ & $28(70 \%)$ & $16(80 \%)$ & $15(75 \%)$ \\
\hline 50 & Glass block rectangle & $39(97.5 \%)$ & $33(82.5 \%)$ & $16(80 \%)$ & $16(80 \%)$ \\
\hline 51 & Hydrometer range & $21(52.5 \%)$ & $18(45 \%)$ & $12(60 \%)$ & $11(55 \%)$ \\
\hline 52 & Hydrometer accumulator & $16(40 \%)$ & $8(20 \%)$ & $4(20 \%)$ & $4(20 \%)$ \\
\hline 53 & Hydraulic pressure demonstrator type & $21(52.5 \%)$ & $6(15 \%)$ & $5(25 \%)$ & $4(20 \%)$ \\
\hline 54 & Hare 's apparatus & $14(35 \%)$ & $8(20 \%)$ & $2(4 \%)$ & $1(5 \%)$ \\
\hline 55 & Hypsometer & $12(30 \%)$ & $7(17.5 \%)$ & $2(4 \%)$ & $2(4 \%)$ \\
\hline 56 & Hope 's apparatus & $12(30 \%)$ & $6(17.5 \%)$ & $1(5 \%)$ & $1(5 \%)$ \\
\hline 57 & Inclined plane and friction board & $26(65 \%)$ & $26(65 \%)$ & $15(75 \%)$ & $14(70 \%)$ \\
\hline 58 & Iron filing (fine) & $33(82.5 \%)$ & $31(77.5 \%)$ & $10(50 \%)$ & $10(50 \%)$ \\
\hline 59 & Induction coil model & $22(55 \%)$ & $22(55 \%)$ & $5(25 \%)$ & $4(20 \%$ \\
\hline 60 & Iron U-bend for electromagnet & $30(75 \%)$ & $30(75 \%)$ & $3(15 \%)$ & $2(4 \%)$ \\
\hline 61 & Jockeys & $34(85 \%)$ & $34(85 \%)$ & $12(60 \%)$ & $6(30 \%)$ \\
\hline 62 & Knife edges & $31(77.5 \%)$ & $30(75 \%)$ & $14(70 \%)$ & $13(65 \%)$ \\
\hline 63 & Kinetic theory model kit & $18(45 \%)$ & $18(45 \%)$ & $3(15 \%)$ & $2(4 \%)$ \\
\hline 64 & Knitting needle & $16(40 \%)$ & $16(40 \%)$ & $10(50 \%)$ & $6(30 \%)$ \\
\hline 65 & Key plugs & $40(100 \%)$ & $38(95 \%)$ & $11(55 \%)$ & $11(55 \%)$ \\
\hline 66 & Linear expansion apparatus & $13(32.5 \%)$ & $11(27.5 \%)$ & $10(50 \%)$ & $10(50 \%)$ \\
\hline 67 & Leslie cube & $12(30 \%)$ & $8(20 \%)$ & $3(15 \%)$ & $3(15 \%)$ \\
\hline 68 & Lenses double convex & $35(87.5 \%)$ & $30(75 \%)$ & $14(70 \%)$ & $14(70 \%)$ \\
\hline 69 & Double concave & $30(75 \%)$ & $30(75 \%)$ & $16(80 \%)$ & $14(70 \%)$ \\
\hline 70 & Lens holder wooden & $33(82.5 \%)$ & $31(77.5 \%)$ & $14(70 \%)$ & $13(65 \%)$ \\
\hline 71 & Leyden jar & $7(17.5 \%)$ & $16(15 \%)$ & $4(20 \%)$ & $4(20 \%)$ \\
\hline 72 & Micrometer screw gauge & $31(77.5 \%)$ & $30(75 \%)$ & $14(70 \%)$ & $13(65 \%)$ \\
\hline 73 & Material kit(solid in rectangle blocks) & $21(52.5 \%)$ & $20(50 \%)$ & $10(50 \%)$ & $10(50 \%)$ \\
\hline 74 & Magnet rectangle $60 \times 15 \times 5 \mathrm{~mm}$ & $18(45 \%)$ & $17(42.5 \%)$ & $11(55 \%)$ & $10(50 \%)$ \\
\hline 75 & Horse shoe & $15(37.5 \%)$ & $14(35 \%)$ & $10(50 \%)$ & $5(25 \%)$ \\
\hline 76 & Magnetizing and demagnetizing coil & $9(22.5 \%)$ & $9(22.5 \%)$ & $5(25 \%)$ & $4(20 \%)$ \\
\hline 77 & Magnetic needles with stands & $14(35 \%)$ & $10(25 \%)$ & $10(50 \%)$ & $5(25 \%)$ \\
\hline 78 & Meter bridge & $33(82.5 \%)$ & $33(82.5 \%)$ & $14(70 \%)$ & $13(65 \%)$ \\
\hline
\end{tabular}




\begin{tabular}{|c|c|c|c|c|c|}
\hline 79 & Measuring cylinders $100 \mathrm{ml}$ & $34(85 \%)$ & $34(85 \%)$ & $16(80 \%)$ & $14(70 \%)$ \\
\hline 80 & Mirrors, cylindrical concave glass & $28(70 \%)$ & $27(67.5 \%)$ & $16(80 \%)$ & $14(70 \%)$ \\
\hline 81 & Spherical concave focus & $24(60 \%)$ & $24(60 \%)$ & $13(65 \%)$ & $11(55 \%)$ \\
\hline 82 & Newton's disc & $15(37.5 \%)$ & $10(25 \%)$ & $3(15 \%)$ & $3(15 \%)$ \\
\hline 83 & Optical screens white & $31(77.5 \%)$ & $31(77.5 \%)$ & $16(80 \%)$ & $12(60 \%)$ \\
\hline 84 & Pulleys & $33(82.5 \%)$ & $33(82.5 \%)$ & $16(80 \%)$ & $12(60 \%)$ \\
\hline 85 & Pendulum & $33(82.5 \%)$ & $33(82.5 \%)$ & $17(85 \%)$ & $13(65 \%)$ \\
\hline 86 & Pumps lift & $15(37.5 \%)$ & $15(37.5 \%)$ & $4(20 \%)$ & $4(20 \%)$ \\
\hline 87 & Bicycle & $5(12.5 \%)$ & $5(12.5 \%)$ & $1(5 \%)$ & $1(5 \%)$ \\
\hline 88 & Pin -optical expt. & $25(62.5 \%)$ & $25(62.5 \%)$ & $16(80 \%)$ & $15(75 \%)$ \\
\hline 89 & Perspex prism & $12(30 \%)$ & $11(27.5 \%)$ & $10(50 \%)$ & $10(50 \%)$ \\
\hline 90 & Plotting compass & $18(45 \%)$ & $18(45 \%)$ & $5(25 \%)$ & $5(25 \%)$ \\
\hline 91 & Plug leads(red and black) length 30cl & $35(87.5 \%)$ & $11(20 \%)$ & $10(50 \%)$ & $10(50 \%)$ \\
\hline 92 & Potentiometer & $37(92.5 \%)$ & $33(82.5 \%)$ & $15(75 \%)$ & $14(70 \%)$ \\
\hline 93 & Pitch box & $5(12.5 \%)$ & $5(12.5 \%)$ & $10(50 \%)$ & $5(25 \%)$ \\
\hline 94 & Power unit( low voltage) & $16(40 \%)$ & $16(40 \%)$ & $10(50 \%)$ & $10(50 \%)$ \\
\hline 95 & Ruler Hard wood & $14(35 \%)$ & $14(35 \%)$ & $10(50 \%)$ & $10(50 \%)$ \\
\hline 96 & Rubber tubing Int. & $13(32.5 \%)$ & $12(30 \%)$ & $4(20 \%)$ & $3(15 \%)$ \\
\hline 97 & Tubbing P.V.C Int. & $12(30 \%)$ & $12(30 \%)$ & $10(50 \%)$ & $5(25 \%)$ \\
\hline 98 & Regnaults hygrometer & $9(22.5 \%)$ & $8(20 \%)$ & $4(20 \%)$ & $4(20 \%)$ \\
\hline 99 & Radiation kit & $12(30 \%)$ & $4(10 \%)$ & $4(20 \%)$ & $4(20 \%)$ \\
\hline 100 & $\begin{array}{l}\text { Ray optics box with accessories } 12 \mathrm{~V} 12 \\
\text { watts bulb }\end{array}$ & $31(77.5 \%)$ & $26(65 \%)$ & $16(80 \%)$ & $14(70 \%)$ \\
\hline 101 & Ray optics and color mixing & $22(55 \%)$ & $22(55 \%)$ & $5(25 \%)$ & $5(25 \%)$ \\
\hline 102 & Refraction and reflection trough & $12(30 \%)$ & $11(27.5 \%)$ & $10(50 \%)$ & $10(50 \%)$ \\
\hline 103 & Ray lamp(for above) & $27(67.5 \%)$ & $21(52.5 \%)$ & $15(75 \%)$ & $13(65 \%)$ \\
\hline 104 & Resonance tube apparatus & $20(50 \%)$ & $18(45 \%)$ & $3(15 \%)$ & $3(15 \%)$ \\
\hline 105 & Ripple tank and accessories & $15(37.5 \%)$ & $15(37.5 \%)$ & $1(5 \%)$ & $1(5 \%)$ \\
\hline 106 & Resistors & $33(82.5 \%)$ & $33(82.5 \%)$ & $16(80 \%)$ & $16(80 \%)$ \\
\hline 107 & Rheostats $0-15$ ohms,4A & $35(87.5 \%)$ & $35(87.5 \%)$ & $15(75 \%)$ & $15(75 \%)$ \\
\hline 108 & Retort stands ,boss lead and clamps & $38(95 \%)$ & $38(95 \%)$ & $15(75 \%)$ & $15(75 \%)$ \\
\hline 109 & Resistance box $0-1000 \mathrm{ohms}$ & $27(67.5 \%)$ & $27(67.5 \%)$ & $16(80 \%)$ & $15(75 \%)$ \\
\hline 110 & Rods mild steel $20 \mathrm{cmx} 1.2 \mathrm{~mm}$-diam. & $6(15 \%)$ & $6(15 \%)$ & $4(20 \%)$ & $4(20 \%)$ \\
\hline 111 & Rods soft iron $1 \mathrm{~cm}$ diam. & $8(20 \%)$ & $9(22.5 \%)$ & $10(50 \%)$ & $9(45 \%)$ \\
\hline 112 & Spring balance & $35(87.5 \%)$ & $35(87.5 \%)$ & $17(85 \%)$ & $16(80 \%)$ \\
\hline 113 & Scale pans for balances & $35(87.5 \%)$ & $26(65 \%)$ & $11(55 \%)$ & $10(50 \%)$ \\
\hline 114 & Sliding vernier calipers & $38(95 \%)$ & $34(85 \%)$ & $13(65 \%)$ & $13(65 \%)$ \\
\hline 115 & Stop watches & $38(95 \%)$ & $38(95 \%)$ & $16(80 \%)$ & $16(80 \%)$ \\
\hline 116 & Stop clocks & $38(95 \%)$ & $30(95 \%)$ & $16(80 \%)$ & $16(80 \%)$ \\
\hline 117 & Spiral springs & $37(92.5 \%)$ & $37(92.5 \%)$ & $15(75 \%)$ & $15(75 \%)$ \\
\hline 118 & Soft wooden blocks & $25(62.5 \%)$ & $24(60 \%)$ & $17(85 \%)$ & $17(85 \%)$ \\
\hline 119 & Syringes & $30(75 \%)$ & $26(65 \%)$ & $11(55 \%)$ & $8(40 \%)$ \\
\hline 120 & Stands for leslie cube & $4(10 \%)$ & $1(2.5 \%)$ & $10(50 \%)$ & $10(50 \%)$ \\
\hline 121 & Set of 'cinemold' filter & $1(2.5 \%)$ & $1(2.5 \%))$ & $4(20 \%)$ & $4(20 \%)$ \\
\hline 122 & Set becks siren & $3(7.5 \%)$ & $8(20 \%)$ & $4(20 \%)$ & $4(20 \%)$ \\
\hline 123 & Sonometer & $10(25 \%)$ & $9(22.5 \%)$ & $5(25 \%)$ & $5(25 \%)$ \\
\hline 124 & Socket (red and black) & $20(50 \%)$ & $16(40 \%)$ & $11(55 \%)$ & $11(55 \%)$ \\
\hline 125 & Slinky springs & $8(20 \%)$ & $8(20 \%)$ & $4(20 \%)$ & $4(20 \%)$ \\
\hline 126 & Ticker timer & $11(27.5 \%)$ & $10(25 \%)$ & $5(25 \%)$ & $5(25 \%)$ \\
\hline 127 & Ticker timer tapes & $12(30 \%)$ & $6(15 \%)$ & $4(20 \%)$ & $4(20 \%)$ \\
\hline 128 & Trolley & $13(32.5 \%)$ & $11(27.5 \%)$ & $5(25 \%)$ & $5(25 \%)$ \\
\hline 129 & Test tube & $35(87.5 \%)$ & $34(85 \%)$ & $16(80 \%)$ & $16(80 \%)$ \\
\hline 130 & Boiling tube & $30(75 \%)$ & $30(75 \%)$ & $18(80 \%)$ & $18(80 \%)$ \\
\hline 131 & Tripod stands & $37(92.5 \%)$ & $27(67.5 \%)$ & $17(85 \%)$ & $17(85 \%)$ \\
\hline 132 & Thermometer mercury glass & $32(80 \%)$ & $32(80 \%)$ & $16(80 \%)$ & $16(80 \%)$ \\
\hline 133 & Wet and dry bulb & $27(67.5 \%)$ & $27(67.5 \%$ & $11(55 \%)$ & $10(50 \%)$ \\
\hline 134 & Thermal conductivity of metal & $14(35 \%)$ & $14(35 \%)$ & $4(20 \%)$ & $14(70 \%)$ \\
\hline 135 & Tuning forks & $30(75 \%)$ & $27(45 \%)$ & $11(55 \%)$ & $10(50 \%)$ \\
\hline 136 & U-tubes & $27(67.5 \%)$ & $18(45 \%)$ & $16(80 \%)$ & $16(80 \%)$ \\
\hline 137 & Unequal expansion of liquid & $14(35 \%)$ & $4(10 \%)$ & $5(25 \%)$ & $5(25 \%)$ \\
\hline
\end{tabular}




\begin{tabular}{|c|c|c|c|c|c|}
\hline & Apparatus & & & & \\
\hline 138 & Voltmeters $05-3 \mathrm{VD} / \mathrm{C}$ & $32(80 \%)$ & $32(80 \%)$ & $16(80 \%)$ & $16(80 \%)$ \\
\hline 139 & Vacuum pum10p & $18(45 \%)$ & $17(42.5 \%)$ & $5(25 \%)$ & $5(25 \%)$ \\
\hline 140 & Wire eureka 2417 S .W.G,26 SWG & $12(30 \%)$ & $12(30 \%)$ & $10(50 \%)$ & $10(50 \%)$ \\
\hline 141 & Copper & $25(62.5 \%)$ & $24(60 \%)$ & $17(85 \%)$ & $17(85 \%)$ \\
\hline 142 & Whirling table & $11(27.5 \%)$ & $9(22.5 \%)$ & $4(20 \%)$ & $1(5 \%)$ \\
\hline 143 & Wire gauge with asbestos & $20(50 \%)$ & $18(45 \%)$ & $10(50 \%)$ & $5(25 \%)$ \\
\hline 144 & Candles & $25(62.5 \%)$ & $25(62.5 \%$ & $14(70 \%)$ & $14(70 \%)$ \\
\hline 145 & Plasticine & $26(65 \%)$ & $26(65 \%)$ & $10(50 \%)$ & $10(50 \%)$ \\
\hline 146 & Cellotape & $28(70 \%)$ & $26(65 \%)$ & $16(80 \%)$ & $16(80 \%)$ \\
\hline 147 & Sealing wax & $17(42.5 \%)$ & $17(42.5 \%)$ & $5(25 \%)$ & $5(25 \%)$ \\
\hline 148 & Screw(assorted size) & $24(60 \%)$ & $23(57.5 \%)$ & $13(65 \%)$ & $13(65 \%)$ \\
\hline 149 & Pliers & $27(67.5 \%)$ & $22(55 \%)$ & $13(65 \%)$ & $13(65 \%)$ \\
\hline 150 & Pincers & $16(40 \%)$ & $10(25 \%)$ & $10(50 \%)$ & $10(50 \%)$ \\
\hline 151 & Wood splint & $15(37.5 \%)$ & $8(20 \%)$ & $10(50 \%)$ & $10(50 \%)$ \\
\hline 152 & Cotton thread & $27(67.5 \%)$ & $27(67.5 \%)$ & $15(75 \%)$ & $15(75 \%)$ \\
\hline 153 & Filter paper & $35(87.5 \%)$ & $35(87.5 \%)$ & $17(85 \%)$ & $17(85 \%)$ \\
\hline 154 & Grease & $28(70 \%)$ & $28(70 \%)$ & $10(50 \%)$ & $10(50 \%)$ \\
\hline 155 & Thin machine oil for lubrication & $27(67.5 \%)$ & $15(37.5 \%)$ & $10(50 \%)$ & $10(50 \%)$ \\
\hline 156 & Lubricating paper & $31(77.5 \%)$ & $31(77.5 \%)$ & $14(70 \%)$ & $13(65 \%)$ \\
\hline 157 & Graph sheet & $31(77.5 \%)$ & $31(77.5 \%)$ & $12(60 \%)$ & $12(60 \%)$ \\
\hline 158 & Paper cartridge & $15(37.5 \%)$ & $15(37.5 \%)$ & $10(50 \%)$ & $10(50 \%)$ \\
\hline 159 & Drawing pins & $37(92.5 \%)$ & $35(87.5 \%)$ & $12(60 \%)$ & $10(50 \%)$ \\
\hline 160 & Soldering iron & $21(52.5 \%)$ & $21(52.5 \%)$ & $10(50 \%)$ & $16(80 \%)$ \\
\hline 161 & Scissors & $32(80 \%)$ & $30(75 \%)$ & $14(70 \%)$ & $14(70 \%)$ \\
\hline 162 & Ammonium chloride & $31(77.5 \%)$ & $31(77.5 \%)$ & $12(60 \%)$ & $10(50 \%)$ \\
\hline 163 & Calcium chlorine & $30(75 \%)$ & $30(75 \%)$ & $14(70 \%)$ & $13(65 \%)$ \\
\hline 164 & Copper Sulphate & $27(67.5 \%)$ & $26(65 \%)$ & $13(65 \%)$ & $13(65 \%)$ \\
\hline 165 & Distilled water & $26(65 \%)$ & $26(65 \%)$ & $13(65 \%)$ & $13(65 \%)$ \\
\hline 166 & Kerosene & $35(87.5 \%)$ & $32(80 \%)$ & $11(55 \%)$ & $11(55 \%)$ \\
\hline 167 & Mercury & $26(65 \%)$ & $26(65 \%)$ & $10(50 \%)$ & $10(50 \%)$ \\
\hline 168 & Methylated spirit & $34(85 \%)$ & $31(77.5 \%)$ & $16(80 \%)$ & $15(75 \%)$ \\
\hline 169 & Nitric acid & $28(70 \%)$ & $28(70 \%)$ & $15(75 \%)$ & $12(60 \%)$ \\
\hline 170 & Potassium hydroxide & $25(62.5 \%)$ & $25(62.5 \%)$ & $18(90 \%)$ & $13(65 \%)$ \\
\hline 171 & Boyle's law apparatus & $9(22.5 \%)$ & $9(22.5 \%)$ & $10(50 \%)$ & $10(50 \%)$ \\
\hline 172 & Brownian motion apparatus & $11(27.5 \%)$ & $11(27.5 \%)$ & $10(50 \%)$ & $10(50 \%)$ \\
\hline 173 & Bar and gauge apparatus & $16(40 \%)$ & $12(30 \%)$ & $10(50 \%)$ & $7(35 \%)$ \\
\hline 174 & Constant volume apparatus & $7(17.5 \%)$ & $7(17.5 \%)$ & $10(50 \%)$ & $10(50 \%)$ \\
\hline 175 & Calipers vernier demonstration type & $28(70 \%)$ & $25(62.5 \%)$ & $15(75 \%)$ & $12(60 \%)$ \\
\hline 176 & Displacement vessel(metal) & $4(10 \%)$ & $4(10 \%)$ & $10(50 \%)$ & $10(50 \%)$ \\
\hline 177 & Equality of pressure in liquid apparatus & $6(15 \%)$ & $6(15 \%)$ & $2(10 \%)$ & $2(10 \%)$ \\
\hline 178 & Hydrometer for universal & $6(15 \%)$ & $5(12.5 \%)$ & $4(20 \%)$ & $4(20 \%)$ \\
\hline 179 & Iron rods-soft iron for electromagnets & $13(32.5 \%)$ & $13(32.5 \%)$ & $12(60 \%)$ & $10(50 \%)$ \\
\hline 180 & $\begin{array}{l}\text { Metal blocks assorted shapes of copper } \\
\text { iron, brass, lead }\end{array}$ & $13(32.5 \%)$ & $7(17.5 \%)$ & $16(80 \%)$ & $10(50 \%)$ \\
\hline 181 & Plane $10 \mathrm{~cm} \times 2.5$ & $8(20 \%)$ & $8(20 \%)$ & $10(50 \%)$ & $10(50 \%)$ \\
\hline 182 & Force & $9(22.5 \%)$ & $8(20 \%)$ & $10(50 \%)$ & $10(50 \%)$ \\
\hline 183 & Rubber bungs, woodcorks-assorted 3 sets & $21(52.5 \%)$ & $20(50 \%)$ & $10(50 \%)$ & $10(50 \%)$ \\
\hline 184 & Slottedweightsetsx100g,2x20g,2x10g & $21(52.5 \%)$ & $21(52.5 \%)$ & $13(65 \%)$ & $11(55 \%)$ \\
\hline 185 & $\begin{array}{l}\text { Sets of assorted rods for assorted metals for } \\
\text { conductivity }\end{array}$ & $17(42.5 \%)$ & $17(42.5 \%)$ & $13(65 \%)$ & $11(55 \% 0$ \\
\hline 186 & Sarvet's toothed wheel & $5(12.5 \%)$ & $5(12.5 \%)$ & $10(50 \%)$ & $10(50 \%)$ \\
\hline 187 & Clinical thermometer & $17(4.5 \%)$ & $13(32.5 \%)$ & $10(50 \%)$ & $10(50 \%)$ \\
\hline 188 & Max and Min thermometer & $13(32.5 \%)$ & $13(32.5 \%)$ & $10(50 \%)$ & $10(50 \%)$ \\
\hline 189 & Turning forks and resonance boxes & $20(50 \%)$ & $17(42.5 \%)$ & $11(55 \%)$ & $10(50 \%)$ \\
\hline 190 & Turning fork of different frequency & $12(30 \%)$ & $10(25 \%)$ & $11(55 \%)$ & $10(50 \%)$ \\
\hline 191 & Triple $0-1.5,3,13 \mathrm{VD} / \mathrm{Cvolt}$ & $14(35 \%)$ & $14(35 \%)$ & $11(55 \%)$ & $10(50 \%)$ \\
\hline 192 & WiresNichrome26,28,32S.WG & $12(30 \%)$ & $9(22.5 \%)$ & $10(50 \%)$ & $4(20 \%)$ \\
\hline 193 & Wiresconstantanbare 24,26,28,32 S.W.G & $26(65 \%)$ & $20(50 \%)$ & $10(50 \%)$ & $10(50 \%)$ \\
\hline
\end{tabular}

$\mathrm{AV}=$ Available, $\mathrm{UT}=$ Utilization 
Table 2 shows the responses of the Physics teachers on the availability and adequacy of laboratory facilities in private and public senior secondary schools in Ondo West Local Government Area in Ondo State of Nigeria. From the 193 items listed on the table it can be seen that only 35 items in public senior secondary schools and 17 items in private senior secondary schools were considered not available because they do not have $50 \%$ of the respondents indicating their availability in schools. Among the unavailable items in both private and public senior secondary schools were compass mariners, dip circle, dip needles, electroscope simple flat type(complete), electric bells, glass cube hallow, fur, flame spectra kit, fluid pressure apparatus ,slinky spring, ticker timer tapes, trolley, hydrometer, accumulator, hare's apparatus, hypsometer, hope's apparatus, kinetic theory model kit, set beck's siren, sonometer, Leslie cube ,Leyden jar ,magnetizing and demagnetizing coil, neutron's disc, pump lift, bicycle, plotting compass, rubber tubing int, thermal conductivity metals, unequal whirling tube, sealing wax, regnauts hygrometer tank and accessories, rod mild steel $20 \mathrm{~cm} \mathrm{x} 1.2 \mathrm{~mm}$ of pressure in liquid apparatus and hydrometer for universal. In addition, induction coil model, iron U-band for electromagnetic, avometer, asbestos sheet $20 \mathrm{~cm} \times 20 \mathrm{~cm}$, aluminum scale pans, balloon, bimetallic strips, breaking bars-cast iron, compass rod, electric motor, electric bell jars, hydraulic pressure- demonstrator type and resonance tube apparatus were not available. While bar and gauge apparatus, glass tube claim, power unit (low voltage), ruler hardwood, tubing pvc int, knitting needle $17 \mathrm{~cm}$, linear apparatus expansion, magnetic rectangle $60 \times 15 \times 15 \mathrm{~mm}$, horse shoe, magnetic needles with stand, Perspex prism pitch balls, refraction and reflection trough, rod soft iron $1 \mathrm{~cm}$, diameter $\mathrm{x}$ $10 \mathrm{~cm}$, stands for leslie cube, wire eureka 24 S.W.G, 26 SWG, pincers, wood split, paper cartridge, Boyle's law apparatus, Brownian motion apparatus, Charles law apparatus displacement vessel (metal block assorted shape for copper iron, brass, lead plane $10 \mathrm{~cm} \times 2.5$, force, sets of assorted rods for assorted metals for conductivity, sarvet's toothed wheel, clinical thermometer, maximum and minimum thermometer, turning fork of different frequencies, triples $0-15,3,30 \mathrm{VD} / \mathrm{C}$ voltmeter and wire nichrome 26, 28, 32 S.W.G were not available in public senior schools. The implication of this result is that, majority of the laboratory facilities for teaching Physics are available in Ondo West Local Government Area in Ondo State of Nigeria public and private senior secondary schools but are not adequate.

Research Questions 3: How utilized are the available laboratory facilities for the teaching of Physics in private and public senior secondary schools in Ondo West Local Government Area in Ondo State?

Table 2 showed that, 103 of the 193 items on Table 2 were considered not utilized in public and private senior secondary schools. Out of 103 items ,59 were not utilized in both public and private senior secondary schools These include balloon, breaking bars, cast iron, compass mariners, compass plotting, dip circle, dip needles, ebonite rod, electroscope simple flat tyre (complete), electric balls, electric bell jars, fur, flame spectra kit, fluid pressure apparatus, glass cube hallow, hydrometer accumulator, hydraulic pressure demonstrator apparatus, hare's apparatus, hypsometer, Hope's apparatus, knitting needle $17 \mathrm{~cm}$, leslie cube, leydenjar, magnetizing and demagnetizing coil, magnetizing needles with stands, Newton's disc, pump lift, bicycle, plotting compass, pitch balls, rubber tubing int, tubing pvc int, regnauts hygrometer, radiation kit, resonance tube apparatus, ripple tank and accessories, rod mild steel $20 \mathrm{cmx}-1.2 \mathrm{~mm}$ - diam, Red soft iron, $1 \mathrm{~cm}$ diam x $10 \mathrm{~cm}$, set of six "cinemoid" filter, set beets siren, senometer, slink springs, ticker timer, ticker timer tapes, trolley unequal expansion of liquid apparatus, whirling table, scaling wax, bar and gauge apparatus, equality of pressure in liquid apparatus, hydrometer for universal wires nchrome 26, 28, 32 SWG. In addition, 33 items were not utilized in public senior secondary schools. These include, bar and gauge apparatus, cells-Daniel complete, glass tube claim, hydrometer range, linear expansion apparatus, magnet rectangle 60x15x15mm, horse shoe, perspex prism, plug leads (red and black) length $30 \mathrm{~cm}$ power unit (10w voltage), Ruler (hardwood), refraction and reflection trough, stand for Leslie cube, socket (red and black), thermal conductivity of metals, U tubes, vacuum pump, wire Eureka 24 SWG, 26 SWG wire guage with asbestos, pincers, wood splint, paper. cartridge, iron rods, soft iron for electromagnets, metal blocks assorted shapes of copper $1 \mathrm{~m}$, brass, lead plane $10 \mathrm{~cm} \times 2.5$, force, sets of assorted rods for assorted metals for conductivity, sarvet's toothed wheel, clinical thermometer, maximum and minimum thermometers, tuning forks on resonance boxes, turning forks of different frequencies and triple $0-1.5,3,30$ VDK voltmeter. avometer, asbestos sheet $20 \mathrm{~cm} \times 20 \mathrm{~cm}$, aluminum scale pans, bimetallic strips cork burners, density bottles, electric meter (1walt) $1-5 \mathrm{~g} 6 \mathrm{v}$, glass block rectangle, induction coil mole) iron $U$ bend for electromagnet, ray optics and colour mixing, and syringes. The implication of this result is that, some of the laboratory facilities are adequate and not 
well utilized in making the state technologically developed. This finding is in agreement with the work of Onabanjo and Akinsola (2000) that, the laboratory facilities available in Oyo State of Nigeria are not adequately utilized. The result is also in agreement with Akinbobola (2006) that, non-human resources in senior secondary schools in Akwa Ibom State are not adequately utilized.

Hypothesis 1: There is no significant difference between the number of qualified Physics teacher in private and public senior secondary schools in Ondo West Local Government Area in Ondo State of Nigeria.

The analysis is shown in Table 3

Table3: Chi square analysis of number of qualified Physics teachers in private and public senior secondary schools

\begin{tabular}{|c|c|c|c|c|c|c|c|}
\hline Qualification & $\begin{array}{l}\text { Public } \\
\text { School }\end{array}$ & $\begin{array}{l}\text { Private } \\
\text { School }\end{array}$ & Row total & df & $\begin{array}{ll}\text { Cal. } & x^{2}- \\
\text { value } & \end{array}$ & $\begin{array}{ll}\text { Crit. } & x^{2}- \\
\text { value } & \end{array}$ & $\begin{array}{l}\text { Decision } \\
\text { at } p<.05\end{array}$ \\
\hline Qualified & 34 & 11 & 45 & 1 & 6.40 & 3.84 & $\mathrm{~S}$ \\
\hline Not qualified & 6 & 9 & 15 & & & & \\
\hline Total column & 40 & 20 & 60 & & & & \\
\hline
\end{tabular}

$\mathrm{S}=$ Significant at $\mathrm{p}<.05$ alpha level

As shown in Table 3, the calculated $\mathrm{x}^{2}$-value of 6.40 is greater than the critical $\mathrm{x}^{2}$-value of 3.84 . Thus, the null hypothesis stating non-significant difference between the number of qualified Physics teachers in private and public senior secondary schools in Ondo West Local Government Area in Ondo State of Nigeria was rejected. The result in Table 3 also indicated that, there are more qualified Physics teachers in public senior secondary schools than private senior secondary schools. This is in line with the findings of Odubunmi and Balogun (1991) that serious, short falls have always existed in the number of professionally qualified science and technical teachers, needed in the nations, schools and colleges. The result is also in agreement with the findings of Akinbobola (2006) that, there are more Physics teachers in public senior secondary schools than private senior secondary schools in Akwa Ibom State of Nigeria.

Hypothesis 2: There is no significant difference between the quantity of available laboratory facilities for teaching Physics in private and public senior secondary schools in Ondo West Local Government Area in Ondo State of Nigeria.

The analysis as shown in Table 4.

Table4: Chi square analysis of laboratory facilities available in private and public senior secondary schools

\begin{tabular}{|l|l|l|l|l|l|l|l|}
\hline Availability & Public School & $\begin{array}{l}\text { Private } \\
\text { School }\end{array}$ & Row total & df & $\begin{array}{l}\text { Cal. } \mathbf{x}^{2}- \\
\text { value }\end{array}$ & $\begin{array}{l}\text { Crit. } \mathbf{x}^{2}- \\
\text { value }\end{array}$ & $\begin{array}{l}\text { Decision } \\
\text { at p<.05 }\end{array}$ \\
\hline Available & $3395(3510.52)$ & $1744(1628.47)$ & 5139 & 1 & 67.89 & 3.84 & $\mathrm{~S}$ \\
\hline Not available & $869(753.48)$ & $234(349.52)$ & 1103 & & & & \\
\hline Total column & 4264 & 1978 & 6242 & & & & \\
\hline
\end{tabular}

$\mathrm{S}=$ Significant at $\mathrm{p}<.05$ alpha level

As shown in Table 4, the calculated $x^{2}$-value 67.89 was greater than the critical value of 3.84.Thus, the null hypothesis which stated that there is no significant difference between the quantity of available laboratory facilities for teaching Physics in private and public senior secondary schools in Ondo West Local Government Area in Ondo State of Nigeria was rejected.

The result in Table 4 also indicated that, the available numbers of laboratory facilities for teaching Physics in public senior secondary schools are more than that of the private senior secondary senior schools. The result is in consistent with the findings of Ajetunmobi and Bashorun in (1999) that science tools, equipment facilities and infrastructures for science teachers are appalling in Nigeria.

Hypothesis 3: There is no significant difference between the utilization of the available laboratory facilities for the teaching of Physics in private and public senior secondary schools in Ondo West Local Government Area in Ondo State of Nigeria.

The analysis is as shown in Table 5. 
Table5: Chi square analysis of utilization of the available laboratory facilities for the teaching of Physics

\begin{tabular}{|l|l|l|l|l|l|l|l|}
\hline Utilization & Public School & $\begin{array}{l}\text { Private } \\
\text { School }\end{array}$ & Row total & df & $\begin{array}{l}\text { Cal. } \mathbf{x}^{2}- \\
\text { value }\end{array}$ & $\begin{array}{l}\text { Crit. } \mathbf{x}^{2}- \\
\text { value }\end{array}$ & $\begin{array}{l}\text { Decision } \\
\text { at p<.05 }\end{array}$ \\
\hline Available & $1598(1451.49)$ & $2920(3066.51)$ & 4518 & 1 & 67.89 & 3.84 & $\mathrm{~S}$ \\
\hline Not available & $266(412.51)$ & $1018(871.49)$ & 1284 & & & & \\
\hline Total column & 1864 & 3938 & 5802 & & & & \\
\hline
\end{tabular}

$\mathrm{S}=$ Significant at $\mathrm{p}<.05$ alpha level

As shown in Table 5, the calculated $\mathrm{x}^{2}$-value of 98.45 is greater than the critical value of 3.84.Thus, the null hypothesis which stated that, there is no significant difference between the utilization of the available laboratory facilities for the teaching of Physics in private and public senior secondary schools in Ondo West Local Government Area in Ondo State of Nigeria was rejected. The result in Table 5 also indicated that, the utilization of the available facilities for teaching Physics in public secondary schools is more than that of private schools.

\section{CONCLUSION}

Based on the study findings, the researchers conclude that:

- Most practical lessons were either ignored or not properly organized to perform an experiment, discuss results and conclude because of inadequate laboratory facilities in both private and public senior secondary schools.

- Apparatus, tools, equipment and materials were used mostly for class demonstrations.

- Physics teachers were unable to teach very well because of inadequate resources.

- Necessity of resources was not taken into consideration from requisitions but instead, urgency and cost were mostly considered.

- Due to free secondary education in Nigeria, high student enrolment affected the provision of adequate resources due to inadequate funds from the government.

- Majority of the Physics teachers available in senior secondary schools in Ondo West Local Government Area in Ondo State, Nigeria are qualified to teach Physics but the numbers are not adequate.

- There are more qualified Physics teachers and laboratory facilities in public senior secondary schools than private senior secondary schools.

- Laboratory facilities for the teaching of Physics in Ondo West Local Government Area in Ondo State, Nigeria are not adequate in number for effective teaching

\section{RECOMMENDATIONS}

Based on the research findings, the following recommendations were made:

- More resources should be mobilized and sent to schools to support and sustain the effective use of the laboratories and direct employment of more Physics teachers to boost the learning process. The available resources within the ministry of education should be relocated within the education subsectors to support the teaching of Physics in secondary schools in order to achieve our vision for quality education.

- Schools depending on the existing circumstances should start alternative and sustainable ways of raising funds for the provision of laboratory facilities. These include Agricultural Practices, use of Parents Teachers Association (PTA) among others. The money realise can be used in the acquisition and maintenance of resources.

- The government alone cannot provide for all the educational needs of her people. More emphasis on cost sharing in the provision of resources is very important. The parents should employ more teachers through PTA in order to compliment those employed by the Teaching Service Commission (TESCOM).

- In order to meet and cater for the needs of the schools, the public schools principals, private schools proprietor and parents should sponsor more Physics teachers to seminars and workshops. 
This will motivate the teachers and inject professionalism, more critical capacity building at all levels and serious planning in the implementation of the curriculum process.

- There is need for a responsive national sensitization and public education programme on free secondary education in Nigeria to sensitize parents and communities about free secondary education. This will clear the notion that currently surrounds free secondary programme as far as cost and financing of the programme is concerned. It is not the responsibility of the government alone with the rising cost of living; the parents must subsidize to help it succeed.

- The quality assurance and standards department should employ more and train staff on how to monitor all the facilities in all secondary schools and advice as well as report to ministry headquarters.

- With the increasing numbers of students in enrolments, the facilities always seem inadequate. The Non-Governmental Organisations (NGOs) and Philanthropist should assist in funding science education in Nigeria.

\section{REFERENCES}

[1] Achimugu, A. (1998). Status of human resources for communicating science, technology and mathematics (STM) education. $39^{\text {th }}$ annual conference proceeding of science teacher association of Nigeria (STAN).

[2] Ajetunmobi, A.W. \& Bashorun, O.W. (1999). Strategies for effective teaching-learning process in agricultural science education at the secondary school level. 40th annual conference proceeding of STAN. pp. 75-79.

[3] Akinbobola, A.O. (2006). Status of resources for physics teaching in public and private secondary schools in Akwa Ibom State. 47th annual conference proceeding of STA. pp. 298-304.

[4] Akinbobola, A.O. (2015). Evaluating science laboratory classroom learning environment in Osun State of Nigeria for national development. Journal of Resources Development and Management, 9, 14-19.

[5] Akinbobola, A.O. \& Bada, A.A. (2017). A compendium of teaching methods. In S.O. Oyekan, A.O. Akinbobola \& R.F. Adebile (Eds.), Creative learning of physics. Ondo: Patrick Ade Prints (Nig.) Ltd.

[6] Akpochafo, W. (2003). Resource management for effective instruction in Nigerian universities: Some practical consideration. Journal of WCCI Nigerian Chapter, 4(1), 66-75.

[7] Hofstein, A. \& Lunetta, V.N. (2004). The laboratory in science education: Foundation for the 21 st century. Science Education, 88, 28-54.

[8] Hofstein, A. \& Mamlok-Naaman, R. (2007). The laboratory in science education: The state of the art. Journal of Chemistry Education Research and Practice, 8(2), 105-107.

[9] Hofstein, A., Navon, O., Kipnis, M. \& Mamlok-Naaman, R. (2005). Developing students' ability to ask more and better questions resulting from inquiry-type chemistry laboratories. Journal of Research in Science Teaching, 40(2), 124-138.

[10] Krajcik, J., Mamlok, R. \& Hug, B. (2001). Modern content and the enterprise of science: Science education in the 20th century. In L. Corno (Rd.), Education across a century” The centennial, pp. 205238. Chicago, Illinois: National Society for the Study of Education.

[11] Molla, T. (2012). Higher education policy reform in Ethiopia: The representation of the problem of gender inequality. Higher Education Policy, 26, 193-215.

[12] Moorhead, G. \& Friffin, R.W. (2002). Organisational behaviour. New York: Houghton Mifflin.

[13] National Research Council (NRS, 2000). Inquiry and the national science education standards. Washington DC: National Academy Press.

[14] Odubunmi, O. \& Balogun, T.A. (1991). The effect of laboratory and lecture teaching methods on cognitive achievement in integrated science. Journal of Research in Science Teaching, 28, 213-224.

[15] Ogweno, P.O. (2015). Teaching and learning resources as determinants of students academic performance in secondary agriculture in Rachuonyo North Sub Country, Kenya. International Journal of Advanced Research, 3(9), 577-587.

[16] Olufunke, B.T. (2012). Effect of availability and utilization of physics laboratory equipment on students' academic achievement in senior secondary school physics. World Journal of Education, 2(5), 1-7.

[17] Onabanjo, I.O. \& Akinsola, O.S. (2000). An investigation into the utilization of the available resources in mathematics classroom. 41st annual conference proceeding of STAN.

[18] Owoeye, J.S. \& Yara, P.O. (2011). School facilities and academic achievement of secondary school agricultural science in Equity State, Nigeria. Asian Social Science, 7(7), 64-74. 
[19] Oyekan, S.O. \& Akinbobola, A.O. (2017). Basic studies is education. In J.O. Adejumo, S.O. Oyekan, R.O. Oloyede \& M. Alfa-Olasunkande (Eds.), Curriculum and instruction. Ondo: Adeyemi College Academic Press.

[20] Sally, T. (2010). The importance of quality education. China Daily: The Johns Hopkins University Press.

[21] Tobin, K.G. (1990). Research on science laboratory activities in pursuit of better questions and answers to improve learning. School Science and Mathematics, 90, 403-418.

Citation: Akinbobola, Akinyemi Olufunminiyi, Bada, Abiodun Adekunle. "Assessment of Resources for Physics Teaching in Nigerian Senior Secondary Schools". International Journal of Humanities Social Sciences and Education (IJHSSE), vol. 6, no.8, 2019, pp. 1-13. doi: http://dx.doi.org/10.20431/2349-0381.0608001.

Copyright: (C) 2019 Authors. This is an open-access article distributed under the terms of the Creative Commons Attribution License, which permits unrestricted use, distribution, and reproduction in any medium, provided the original author and source are credited. 